\title{
A-DIPLOME ERFREUEN SICH GROSSER BELIEBTHEIT
}

\section{Liebe Kolleginnen, liebe Kollegen!}

Auch dieses Jahr gab es wieder einen Refresher- und Prüfungsvorbereitungskurs mit abschließender A-DiplomPrüfung. „In entspannter Atmosphäre sich ganz auf Akupunktur einlassen“ war auch dieses Mal das Motto derTage an der Mecklenburgischen Seenplatte. Wenn der Kopf aufgrund der vielen Informationen rauchte, ließen sich einige der Teilnehmer einfach mal bei einer Bootsfahrt oder einem Uferspaziergang den Wind um die Ohren fegen und schon fiel das Lernen viel leichter. Viel Lob ernteten die Referenten für ihren Einsatz. Mit großer Geduld und Empathie wurden die Fragen derTeilnehmer beantwortet. So lösten sich dann auch die meisten Verständnisschwierigkeiten in Luft auf. Das A-Diplom ist der Nachweis der abgeschlossenen Basisausbildung und dient als Nachweis der Befähigung gegenüber Patienten und Kostenträgern. Es ist auch die Voraussetzung für weiterführende Diplome und besonders als Vorprüfung für die Zusatzbezeichnung Akupunktur geeignet. Die nächste Möglichkeit, das A-Diplom mit Prüfungsvorbereitungskurs zu absolvieren, ist am 24.05.2020 in Garmisch-Partenkirchen. Außerdem finden in dieser Zeit (21.-27.05.2020) auch die Kurse TCM 11 und 12 für das TCM-Diplom statt. Ersatzweise kann - allerdings ohne Vorbereitungskurs - das A-Diplom auch während des Timmendorf-Kongresses abgelegt werden.

Timmendorf 2019 rückt näher! Es wartet ein breit gefächertes Programm mit Referenten aus dem In- und Ausland zu Themen der Wandlungsphase Holz auf Sie. Schauen Sie ins Programm (www.akupunktur.de | Klick auf den orangenen Button) und melden Sie sich rechtzeitig an, denn die Hotelplätze sind begrenzt. Das begleitende Kongressprogramm mit neuen Workshops wird Ihnen viel Freude bereiten und der Gala-Abend, der dieses Jahr

\section{Die DAA-B informiert:}

Die Bundesärztekammer hat zu einer Auftaktveranstaltung im Juni und zu Bewertungsgesprächen im Juli dieses Jahres die beteiligten ärztlichen Berufsverbände und medizinischwissenschaftlichen Fachgesellschaften eingeladen. Es sind insgesamt 44 Sitzungstermine von Mitte Juni bis Mitte September vorgesehen. Ziel ist es, die Bewertungsergebnisse kritisch zu prüfen und eine Folgenabschätzung vorzunehmen. Die Novellierung der GOÄ geht voran und die DAA e.V.bereitet Sie sowohl mit dem A-Diplom und dem TCM-Diplom als auch mit den Spezial-Diplomen für Störherddiagnostik und C-Diplom auf diese neue Abrechnungsstruktur vor. Es ist davon auszugehen, dass die Kostenträger die „Qualifikationen“, die für die neuen Ziffern erforderlich sind, überprüfen werden.

Mit guten Wünschen für den Sommer

Ihr

B. Ramme

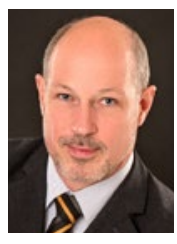

Dr. med. Bernd Ramme

1. Vorsitzender der DAA e.V.

Osserstraße 40, D-81679 München

Tel. $+4989 / 8145252$

E-Mail des allg.Büros: kontakt@akupunktur.de, Internet:www.akupunktur.de 